\title{
White Matter Hyperintensities and the Course of Depressive Symptoms in Elderly People with Mild Dementia
}

\author{
Hogne Soennesyn ${ }^{a} \quad K^{2}$ til Oppedal ${ }^{\mathrm{a}, \mathrm{e}}$ Ole Jacob Greve ${ }^{\mathrm{b}}$ \\ Friederike Fritze $^{c}$ Bjørn H. Auestad ${ }^{f}$ Sabine P. Nore ${ }^{g}$ \\ Mona K. Beyer ${ }^{a, d}$ Dag Aarsland ${ }^{a}$
}

${ }^{a}$ Centre for Age-Related Medicine, ${ }^{b}$ Department of Radiology and ${ }^{\mathrm{C}}$ Department of Geriatric Psychiatry, Psychiatric Clinic, dThe Norwegian Center for Movement Disorders, Stavanger

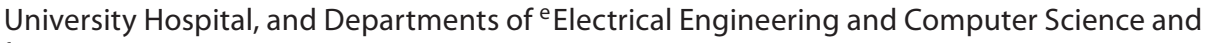
${ }^{\mathrm{f}}$ Mathematics and Natural Sciences, University of Stavanger, Stavanger, and ${ }^{9}$ Section of Geriatric Medicine, Haraldsplass Deaconess University Hospital, Bergen, Norway

\section{Key Words}

White matter hyperintensities $\cdot$ Depression $\cdot$ Depressive symptoms $\cdot$ Dementia $\cdot$ Elderly people

\begin{abstract}
Objectives: To explore the relationship between white matter hyperintensities $(\mathrm{WMH})$ and the prevalence and course of depressive symptoms in mild Alzheimer's disease (AD) and Lewy body dementia. Design: This is a prospective cohort study conducted in secondary care outpatient clinics in western Norway. Subjects: The study population consisted of 77 elderly people with mild dementia diagnosed according to standardised criteria. Methods: Structured clinical interviews and physical, neurological, psychiatric, and neuropsychological examinations were performed and routine blood tests were taken. Depression was assessed using the depression subitem of the Neuropsychiatric Inventory and the Montgomery-Åsberg Depression Rating Scale (MADRS). A standardised protocol for magnetic resonance imaging scan was used, and the volumes of WMH were quantified using an automated method, followed by manual editing. Results: The volumes of total and frontal deep WMH were significantly and positively corre-
\end{abstract}

A previous version was presented in part at the International Psychogeriatrics Association 15th Congress, 8 September, 2011, The Hague, The Netherlands.

Hogne Soennesyn

Centre for Age-Related Medicine

Stavanger University Hospital, PO Box 8100

NO-4068 Stavanger (Norway)

Tel. +47 5151 3436, E-Mail hogne.soennesyn@sus.no 
lated with baseline severity of depressive symptoms, and depressed patients had significantly higher volumes of total and frontal deep WMH than non-depressed patients. Higher volumes of WMH were also associated with having a high MADRS score and incident and persistent depression at follow-up. After adjustment for potential confounders, frontal deep WMH, in addition to prior depression and non-AD dementia, were still significantly associated with baseline depressive symptoms ( $p=0.015$, OR $3.703,95 \% \mathrm{Cl} 1.294-10.593)$. Similar results emerged for total WMH. Conclusion: In elderly people with mild dementia, volumes of $\mathrm{WMH}$, in particular frontal deep $\mathrm{WMH}$, were positively correlated with baseline severity of depressive symptoms, and seemed to be associated with persistent and incident depression at follow-up. Further studies of the mechanisms that determine the course of depression in mild dementia are needed.

Copyright $\odot 2012$ S. Karger AG, Base

\section{Introduction}

White matter hyperintensities (WMH) are commonly found in cerebral T2-weighted magnetic resonance imaging (MRI) scans of elderly people. They seem to be particularly common in depression [1] and dementia [2,3]. Etiologically, they have been associated with classic cardiovascular risk factors [2], including hypertension [4], and thus are considered to be a marker of cerebrovascular disease.

In previous studies of elderly people, the severity of WMH has been found to predict worsening of voiding, mobility and cognition [5], as well as incident depression and depressive symptoms [6,7]. The volume of WMH was associated with incident depression in a study of non-institutionalised elderly people [7], and severe deep white matter lesions predicted poor outcome in elderly patients with major depressive disorder [8].

In subjects with dementia, frontal white matter lesions have been found to be associated with higher depression scores in cross-sectional studies [3, 9]. However, a subsequent study failed to identify an association between WMH and psychiatric or other clinical variables in patients with Alzheimer's disease (AD), using a visual scale [10]. Thus, the implications of WMH with regard to incidence and prognosis of depression in subjects with mild dementia are still unclear. This is unfortunate, since depression is common in dementia [11], and is associated with poorer outcome in terms of cognitive ability [12], functioning [13], and quality of life [14].

The aim of our study was to explore the relationship between WMH and the longitudinal course of depressive symptoms in patients with mild dementia. We hypothesised that prevalence, incidence and prognosis of depressive symptoms are related to the volume of $\mathrm{WMH}$ and frontal deep WMH in particular. Such knowledge might enable a more accurate prognosis, but also increase our understanding of the underlying aetiologies of depression in dementia, and, most importantly, provide a strategy for potential prevention and treatment of depression in dementia.

We have previously [15] reported that depression is more common in dementia with Lewy bodies (DLB) compared to $\mathrm{AD}$, and that $\mathrm{WMH}$ are more severe in mild $\mathrm{AD}$ and DLB patients than in non-demented elderly, using a novel semi-automatic volumetric analysis technique (Ketil Oppedal, Alzheimer's Association International Conference 2011). A secondary aim was, therefore, to explore the association between volumetrically assessed WMH and depression in patients with Lewy body dementia (LBD) and AD separately. 


\section{Methods}

\section{Subjects}

We screened all referrals to the five outpatient clinics in geriatric medicine and old age psychiatry in the counties of Rogaland (Stavanger and Haugesund) and Hordaland (Bergen) in western Norway from March 2005 to March 2007 for patients with a first time diagnosis of mild dementia, i.e. a minimum Mini-Mental State Examination (MMSE) score of 20. From April 2007, we selectively recruited patients with DLB and Parkinson's disease with dementia (PDD). Additionally, three neurology outpatient clinics in the same area were contacted, and agreed to refer new dementia cases to one of the participating centres. The patients and caregivers were first seen by the study clinician, who performed a structured clinical interview of demographic and clinical data. The comprehensive assessment procedure included a detailed history using a semi-structured interview, clinical examination, including physical, neurological, psychiatric, and neuropsychological examinations, and routine blood tests. A total of 215 subjects fulfilled the inclusion criteria and constituted the baseline population. Patients were followed annually with the same assessment battery.

\section{Ethical Issues}

The study was approved by the regional Ethics Committee and the Norwegian authorities for collection of medical data. The patients provided written consent to participate in the study after the study procedures had been explained in detail to the patient and a caregiver, usually the spouse or offspring.

\section{Dementia Diagnosis}

Diagnoses were made after a detailed assessment as previously described [16], including the use of standardised clinical assessments of psychiatric symptoms, including hallucinations and depression, parkinsonism and cognitive fluctuations, and MRI and blood tests. Cerebrospinal fluid was analysed in a subgroup of patients, and most patients with suspected DLB completed a dopamine transporter SPECT scan. Patients with acute delirium, terminal illness, recently diagnosed with a major somatic illness, previous bipolar disorder or psychotic disorder were excluded.

Two of the investigators independently applied the diagnostic criteria after baseline and 2 years later. In cases of disagreement, and in patients fulfilling more than one set of operationalised diagnostic criteria, the final ascertainment was made based on consensus. In our present study, DLB and PDD were combined into one group (LBD), because these conditions have several clinical and biological similarities $[17,18]$.

To determine apolipoprotein E (APOE) genotypes, genomic DNA was extracted from $200 \mu$ l EDTA blood using the QIAamp 96 DNA Blood Kit (Qiagen, Hilden, Germany). The APOE $\varepsilon 2, \varepsilon 3$ and $\varepsilon 4$ genotypes, which are determined by the combination of two SNPs (rs7412 and rs429358), were detected using the LightCycler APOE Mutation Detection Kit (Roche Diagnostics, Mannheim, Germany). The assay was used according to the manufacturer's instructions.

\section{Assessment of Depression}

Depression was assessed using the depression subitem of the Neuropsychiatric Inventory (NPId) and the Montgomery-Åsberg Depression Rating Scale (MADRS), and defined as previously described [15]. The NPI was specifically designed to assess psychiatric symptoms in subjects with dementia, based on a structured interview of a caregiver [19], and the Norwegian version has been validated [20]. It consists of 12 items, including a depression (dysphoria) item (NPId), with a maximum score of 12 . A cutoff score of $\geq 1$ was used 
to detect any depression, and a cutoff score of $\geq 4$ was used for clinically relevant depression [21].

MADRS [22] is a clinical interview with 10 items, each scored from 0 to 6 , and was completed by an experienced licensed geriatrician or psychiatrist, after a training procedure. Raters met biannually to ensure similar administration of the instruments. A cutoff score of $\geq 7$ was used to detect at least mild depression [23], and a cutoff of $\geq 15$ for clinically significant depression [24]. MADRS has been found to provide a good measure of depression relatively independent from dementia severity [25]. A history of clinically relevant (i.e. needing therapy) depression was asked for in the interview. Depression was assessed at baseline and at a follow-up evaluation 1 year later. Based on the MADRS cutoff scores, depression status was classified as never depressed, incident depression, persistent depression or recovered. The same procedure was performed based on the NPId cutoff scores.

\section{Assessment of Physical Comorbidity}

For assessment of physical comorbidity, we employed the Cumulative Illness Rating Scale (CIRS), which measures the chronic medical illness burden, while taking into account the severity of chronic diseases. It was scored by a licensed and experienced geriatrician (S.P.N.) in accordance with the guidelines [26].

\section{MRI}

The subjects were scanned at three different sites: the Stavanger University Hospital, the Haugesund Hospital, and the Haraldsplass Deaconess Hospital (Bergen). A 1.5-T scanner was used in all three centres (Philips Intera in Stavanger and Haugesund, and a 1.5T GE Signa Excite scanner in Bergen). MRI was done with the same scanner in each centre during the entire study period, and a common study imaging protocol was used. In some cases, scans were excluded due to insufficient image quality, not having both FLAIR and T1 images for the patient, or movement artefacts or other artefacts.

\section{Volumetric Assessment of WMH}

3D T1-Weighted Sequence

Stavanger. Repetition time (TR)/echo time (TE) 10.0/4.6 ms, flip angle 30.0 $0^{\circ}$ 2-mm slice thickness with $1 \mathrm{~mm}$ spacing between the slices (1-mm slices with no gap), number of excitations (NEX) 2, Matrix $256 \times 256$, field of view (FOV) $26 \mathrm{~cm}$.

Haugesund. TR/TE 20.0/4.6 ms, flip angle $30.0^{\circ}, 1-\mathrm{mm}$ slice thickness with $1 \mathrm{~mm}$ spacing between the slices, NEX 1, Matrix $512 \times 512$, FOV $26 \mathrm{~cm}$.

Bergen. TR/TE 8.2/3.1 ms, flip angle $7^{\circ}$, 1-mm slice thickness with $1 \mathrm{~mm}$ spacing between the slices, inversion time (TI) $500 \mathrm{~ms}$, NEX 1, Matrix $256 \times 256$, FOV $25.6 \mathrm{~cm}$.

FLAIR Sequence

Stavanger. TR/TE 6,000/100 ms, TI 2,000 ms, 4-mm slice thickness/1-mm gap, NEX 2, Matrix $256 \times 256$.

Haugesund. TR/TE/TI 6,000/110/2,000 ms, 4-mm slice thickness/1-mm gap, NEX 2, Matrix $512 \times 512$.

Bergen. TR/TE 7,927/105.4 ms, flip angle $90^{\circ}$, 4-mm slice thickness/1-mm gap, TI 1,981 ms, NEX 1, Matrix $256 \times 256$.

Processing and Analysis of 3D T1-Weighted Scans and Axial FLAIR Images

Image analysis was performed according to a method developed and previously published by Firbank et al. [27]. This method requires sets of 3D T1-weighted scans and axial FLAIR images. 
The non-brain regions were removed from the T1 image, using the segmentation routines in SPM5 (http://www.fil.ion.ucl.ac.uk/spm), and the WMH were segmented on a sliceby-slice basis from the FLAIR image, with the images in native space, using a threshold determined from the histogram of pixel intensities for each image slice.

To explore the regional distribution of WMH throughout the brain, a region of interest (ROI) template in standard MNI space was used (Montreal Neurological Institute, http:// www.bic.mni.mcgill.ca). This ROI template was transformed from MNI space to the image space (FLAIR) of each subject by use of the normalization routines in SPM5, and the volumes of WMH in each ROI were calculated. The ROI map was based on the Brodmann template, which is provided as part of MRICro (http://www.psychology.nottingham.ac.uk/staff/crl/ lesion.html\#brod). Further details can be found in the study by Firbank et al. [28].

Because of the variability in image quality from the different centres participating in this study, we found it difficult to empirically choose a single threshold level that gave us good enough segmentation results in each subject, as deemed by a qualified radiologist (M.K.B.). A threshold level of 1.2 was chosen, since it gave an overestimation of the lesion load in every subject. Manual editing was then done to correct for this, by removing excess pixels using FSLView (http://www.fmrib.ox.ac.uk/fsl/index.html).

A specialist in internal medicine and geriatrics (H.S.) did the manual editing, blind to clinical data, after training by a consultant neuroradiologist (M.K.B.). They both edited the same 10 datasets twice; once at the beginning, to ensure good interrater reliability, and a second time at the end to ensure that similar reliability still persisted and to evaluate intrarater reliability. The intraclass correlation coefficient was calculated to be 0.998 for interrater reliability and 0.964 for intrarater reliability. The manually edited scans were then used in the further analysis of volumes of total and regional WMH. In order to compensate for interindividual differences in total brain volumes, so as to be able to make comparisons between individual patients, we calculated the ratios of volumes of WMH to total brain volumes, which were then used in the statistical analyses.

Visual Assessment of WMH

MRIs were also rated visually, using the Scheltens scale [29], by an experienced radiologist (O.J.G.) blind to clinical data. Interrater reliability with another neuroradiologist (M.K.B.) was analysed, based on 12 scans, finding an intraclass correlation coefficient of 0.923 .

\section{Statistics}

Baseline comparisons were made using the independent-samples $t$ test, the $\chi^{2}$ test or Fisher's exact test, as appropriate. Depression scores for subjects with and without volumetric MRI were compared using the independent-samples t test or the Mann-Whitney U test, as appropriate. Patients with and without depression were compared using the $\chi^{2}$ test, the independent-samples $t$ test or the Mann-Whitney $U$ test, as appropriate. Spearman's rank order correlations between WMH ratios, Scheltens scores and depression measures, including change between baseline and follow-up, were performed. WMH ratios and Scheltens scores in the various depression status groups at baseline and follow-up were compared using the Mann-Whitney U test. Analysis of covariance (ANCOVA) was performed to control for effects of centre affiliation and other potential confounders. $p$ values $<0.05$ (two-tailed) were considered statistically significant. Potential predictor variables were included in stepwise multiple logistic regression analyses together with frontal deep WMH ratios or total $\mathrm{WMH}$ ratios, using the various definitions of depression as response variable. For these analyses, the frontal deep and total WMH ratios were multiplied by 100 due to very small absolute values. 
Subjects with missing data for a given analysis were excluded from that specific analysis. For determination of the normality status of continuous variables, we used the Kolmogorov-Smirnov test. All statistical tests were performed using PASW Statistics 18, release 18.0.1.

\section{Results}

Of the 215 participants included at baseline, 9 were excluded later. Of these, 5 were rediagnosed as having mild cognitive impairment, 2 withdrew from the study and 2 were excluded due to lack of complete data. A total of 190 of the 206 (92\%) remaining participants completed the 1-year follow-up examination. Death was the major reason for dropout, as 10 died during the first year follow-up period. The other reasons for not being seen at the 1-year follow-up were patient refusal $(n=5)$ or caregiver refusal $(n=1)$.

MRI scans of 77 participants were of sufficient quality for volumetric WMH analysis, and 137 scans could be rated semiquantitatively according to the Scheltens scale. The correlations between the WMH (total and deep frontal) ratios and the Scheltens (total and frontal) scores were highly significant (Spearman's rho 0.838 and 0.839 , respectively, $\mathrm{p}<0.001$ ).

Among the patients with scans included in the volumetric analyses ('completers'), there was a significantly higher proportion of subjects with $\mathrm{AD}$ and a lower proportion with DLB/ PDD, and they had a somewhat shorter mean disease duration than patients who were not included in the volumetric analyses ('non-completers'). Otherwise, there were no significant differences between the 77 completers and the 129 non-completers in demographic and clinical variables at baseline (table 1).

There were no significant differences between the 137 subjects with scans rated with the Scheltens scale and the rest of the subjects $(n=69)$ with regard to baseline clinical characteristics (data not shown).

At baseline, 33 of the 77 patients included in the volumetric analyses were depressed and 44 were not depressed, using a MADRS cutoff score of $\geq 7$. There were no significant differences between depressed and non-depressed patients with regard to sex, use of psychoactive medications, MMSE scores, CIRS total scores, and APOE $\varepsilon 4$ at baseline, but the depressed were significantly younger and included significantly more patients with prior depression and patients with non-AD dementia (table 2). However, the significance of the age difference disappeared after controlling for centre affiliation (data not shown).

Using a MADRS cutoff score of $\geq 15,14$ were depressed and 63 not depressed. The only significant differences regarding the above-mentioned variables were that the depressed significantly more often had also prior depression $(9 / 14 \mathrm{vs.} 13 / 63, \mathrm{p}=0.003)$, and significantly more often were using antidepressants $(8 / 14$ vs. $15 / 63, p=0.024)$.

At the 1-year follow-up, there were no significant differences in the proportions of the depressed and non-depressed receiving antidepressants (MADRS cutoff $\geq 7 ; 13 / 24$ vs. 13/33, $\mathrm{p}=0.403$, MADRS cutoff $\geq 15 ; 3 / 5$ vs. 23/52, $\mathrm{p}=0.651$ ).

\section{Baseline Analyses}

At baseline, significant positive correlations with MADRS scores were found for both total (Spearman's correlation coefficient $0.274, \mathrm{p}=0.016$ ) and frontal deep WMH ratios (Spearman's correlation coefficient $0.238, \mathrm{p}=0.037$ ), and patients with MADRS scores of $\geq 7$ had significantly higher total and frontal deep WMH ratios than patients with MADRS scores of $<7$ ( $p=0.011$ and $p=0.023$, respectively). Similarly, patients with MADRS scores of $\geq 15$ had higher total and frontal deep WMH ratios $(\mathrm{p}=0.018$ and $\mathrm{p}=0.015$, respectively) than those with MADRS scores of 14 or lower. 
Table 1. Baseline clinical characteristics of subjects who participated in the volumetric MRI study (completers) and those who did not participate (non-completers)

\begin{tabular}{lcccc}
\hline & $\begin{array}{l}\text { Non-completers } \\
(\mathrm{n}=129)\end{array}$ & $\begin{array}{l}\text { Completers } \\
(\mathrm{n}=77)\end{array}$ & $\mathrm{p}$ value & $\begin{array}{c}\text { Participants } \\
\text { missing data } \\
(\mathrm{n}=206)\end{array}$ \\
\hline Age & $77.0(71-82)$ & $76.9(70-81)$ & 0.613 & 0 \\
Women & $72(55.8)$ & $50(64.9)$ & 0.195 & 0 \\
Years of education & $8.5(7-11)$ & $8.5(7-11)$ & 0.613 & 27 \\
MMSE & $23(22-26)$ & $24(23-26)$ & 0.230 & 4 \\
AD & $74(57.4)$ & $59(76.6)$ & $\mathbf{0 . 0 0 5}$ & 0 \\
DLB/PDD & $42(32.6)$ & $12(15.6)$ & $\mathbf{0 . 0 0 7}$ & 0 \\
VaD & $9(7)$ & $2(2.6)$ & 0.216 & 0 \\
FTD/alcoholic dementia & $4(3.1)$ & $4(5.2)$ & 0.475 & 0 \\
Mean disease duration (min-max), years & $2.9(0.5-15)$ & $2.3(0.5-12)$ & $\mathbf{0 . 0 4 5}$ & 13 \\
Prior depression & $49(40)$ & $22(29)$ & 0.160 & 7 \\
CIRS total & $6(4-8)$ & $6(4-7)$ & 0.311 & 2 \\
Use of antipsychotics & $5(3.9)$ & $3(3.9)$ & 1.000 & 3 \\
Use of anxiolytics/hypnotics & $20(15.6)$ & $8(10.5)$ & 0.289 & 2 \\
Use of antidepressants & $43(33.6)$ & $23(30.3)$ & 0.625 & 2 \\
MADRS & $8(3.3-12.8)$ & $5(3-12.5)$ & 0.446 & 1 \\
MADRS $\geq 7$ & $70(55)$ & $33(43)$ & 0.135 & 1 \\
MADRS $\geq 15$ & $21(16)$ & $14(18)$ & 0.892 & 1 \\
APOE $4 \geq 1$ allele, fraction $(\%)$ & $58 / 95(61)$ & $32 / 51(63)$ & 0.842 & 111 \\
\hline
\end{tabular}

Values are median (IQR) or $\mathrm{n}(\%)$, unless otherwise indicated.

$\mathrm{VaD}=$ Vascular dementia; $\mathrm{FTD}$ = frontotemporal dementia; MMSE: normal range 24-30; CIRS: range 0 (no impairment)-52 (extremely severe impairment); MADRS: normal range 0-6.

Significant results are shown in bold.

Table 2. Baseline clinical characteristics of non-depressed versus depressed patients (according to the MADRS score)

\begin{tabular}{lcll}
\hline & $\begin{array}{l}\text { MADRS baseline }<7 \\
(\mathrm{n}=44)\end{array}$ & $\begin{array}{l}\text { MADRS baseline } \geq 7 \\
(\mathrm{n}=33)\end{array}$ & $\mathrm{p}$ value \\
\hline Age & $78(71-81)$ & $73(68-80)$ & $\mathbf{0 . 0 4 9 ^ { * }}$ \\
Women & $29(66)$ & $21(64)$ & 0.839 \\
Prior depression & $8(18)$ & $14(44)$ & $\mathbf{0 . 0 3 0}$ \\
Use of antidepressant & $11(25)$ & $12(36)$ & 0.358 \\
Use of anxiolytic/hypnotic & $2(5)$ & $6(19)$ & 0.063 \\
Use of antipsychotic & $2(5)$ & $1(3)$ & 1.000 \\
CIRS total & $6(4-7)$ & $6(3.5-7.5)$ & 0.769 \\
MMSE & $24(22-25)$ & $24(23-26)$ & 0.268 \\
Dementia diagnosis (non-AD) & $5(11)$ & $13(39)$ & $\mathbf{0 . 0 0 7}$ \\
APOE $4 \geq 1$ allele, fraction $(\%)$ & $17 / 28(61)$ & $15 / 23(65)$ & 0.968 \\
\hline
\end{tabular}

Values are median (IQR) or $\mathrm{n}(\%)$, unless otherwise indicated.

MADRS: normal range 0-6; CIRS: range 0 (no impairment)-52 (extremely severe impairment); MMSE: normal range 24-30.

* Not significant after controlling for centre affiliation. Significant results are shown in bold. 
Table 3a. Multiple logistic regression analysis of the effect of frontal deep WMH volume on the likelihood of a MADRS score of $\geq 7$ at baseline

\begin{tabular}{lrrll}
\hline & B & SE & p & \multicolumn{1}{l}{$\begin{array}{l}\text { Odds ratio exp. (B) } \\
\text { (95\% CI) }\end{array}$} \\
\hline Frontal deep WMH ratio & 1.309 & 0.536 & 0.015 & $3.703(1.294-10.593)$ \\
Non-AD versus AD* & -2.055 & 0.666 & 0.002 & $0.128(0.035-0.472)$ \\
Prior depression & 1.321 & 0.611 & 0.031 & $3.746(1.130-12.414)$ \\
\hline
\end{tabular}

Table 3b. Multiple logistic regression analysis of the effect of total WMH volume on the likelihood of a MADRS score of $\geq 7$ at baseline

\begin{tabular}{|c|c|c|c|c|}
\hline & B & SE & $\mathrm{p}$ & $\begin{array}{l}\text { Odds ratio exp. (B) } \\
(95 \% \mathrm{CI})\end{array}$ \\
\hline Total WMH ratio & 0.328 & 0.124 & 0.008 & $1.388(1.088-1.771)$ \\
\hline Non-AD versus $A^{*}$ & -1.841 & 0.656 & 0.005 & $0.159(0.044-0.574)$ \\
\hline Prior depression & 1.300 & 0.605 & 0.031 & $3.671(1.122-12.006)$ \\
\hline
\end{tabular}

* Non-AD $=0, \mathrm{AD}=1$. MADRS: normal range $0-6$.

In contrast, there were no significant correlations between baseline NPId and total and frontal WMH ratios ( $\mathrm{p}=0.163$ and 0.149 , respectively). However, when using a NPId depression cutoff score of $\geq 4$, the depressed $(n=16 / 75)$ had significantly higher total and frontal deep $\mathrm{WMH}$ ratios $(\mathrm{p}=0.010$ and $\mathrm{p}=0.019$, respectively). No significant differences were found using a NPId depression cutoff score of $\geq 1$.

When analysing AD and LBD patients separately, a trend towards a significant positive correlation was found between frontal deep WMH ratios and baseline MADRS scores in AD patients (Spearman's correlation coefficient $0.254, \mathrm{p}=0.052$ ), but not in LBD patients (Spearman's correlation coefficient $0.467, \mathrm{p}=0.126$ ). Total $\mathrm{WMH}$ ratios did not differ between AD or LBD patients according to depression (data not shown).

No significant associations were found between Scheltens total and frontal scores and the depression measures at baseline, except that patients with a NPId score of $\geq 4$ had significantly higher Scheltens deep frontal scores $(\mathrm{p}=0.034)$ than those with a NPId score of $<4$.

We performed stepwise multiple logistic regression in order to assess the potential confounding effects and relative contributions of variables other than total and frontal deep $\mathrm{WMH}$ on the odds of having a MADRS score of $\geq 7$ at baseline.

Due to high correlation between total and frontal deep WMH (correlation coefficient $0.797, \mathrm{p}<0.001$ ), we performed separate analyses for these variables. The initial models contained, in addition to the $\mathrm{WMH}$ variable, five other independent variables (non-AD vs. AD, age at baseline, sex, CIRS total score and prior depression), all of which have been associated with depression in previous studies [15, 30-33].

The final model concerning frontal deep WMH was statistically significant (Omnibus test of model coefficients $\mathrm{p}<0.0005$ ). Frontal deep WMH, in addition to prior depression and type of dementia, were still significantly associated with a baseline MADRS score of $\geq 7$ (table 3a).

Similar results emerged for total WMH. The final model was statistically significant (Omnibus test of model coefficients $\mathrm{p}<0.0005)$, and total WMH were significantly associated with a baseline MADRS score of $\geq 7$ (table $3 b$ ).

Due to the low numbers of patients having clinically significant depression (i.e. MADRS $\geq 15$ or NPId $\geq 4$ ) at baseline and at follow-up (MADRS $n=5 / 65$ and NPId $n=16 / 67$ ), only 
Table 4a. Multiple logistic regression analysis of the effect of frontal deep WMH volume on the likelihood of a MADRS score of $\geq 15$ at baseline

\begin{tabular}{llllll}
\hline & B & SE & $p$ & $\begin{array}{l}\text { Odds ratio exp. (B) } \\
(95 \% \text { CI })\end{array}$ \\
\hline $\begin{array}{lllll}\text { Frontal deep WMH ratio } \\
\text { Prior depression }\end{array}$ & 0.855 & 0.379 & 0.024 & $2.350(1.117-4.943)$ \\
\hline
\end{tabular}

Table 4b. Multiple logistic regression analysis of the effect of frontal deep WMH volume on the likelihood of a MADRS score of $\geq 15$ at baseline

\begin{tabular}{|c|c|c|c|c|}
\hline & B & SE & $\mathrm{p}$ & $\begin{array}{l}\text { Odds ratio exp. (B) } \\
(95 \% \text { CI })\end{array}$ \\
\hline Frontal deep WMH ratio & 0.985 & 0.388 & 0.011 & $2.678(1.251-5.733)$ \\
\hline Non- $A D$ versus $A D^{*}$ & -1.255 & 0.682 & 0.066 & $0.285(0.075-1.085)$ \\
\hline
\end{tabular}

Table 4c. Multiple logistic regression analysis of the effect of total WMH volume on the likelihood of a MADRS score of $\geq 15$ at baseline

\begin{tabular}{llllll}
\hline & B & SE & $p$ & $\begin{array}{l}\text { Odds ratio exp. (B) } \\
\text { (95\% CI) }\end{array}$ \\
\hline $\begin{array}{l}\text { Total WMH ratio } \\
\text { Prior depression }\end{array}$ & 0.225 & 0.113 & 0.046 & $1.252(1.004-1.562)$ \\
& 1.781 & 0.663 & 0.007 & $5.939(1.620-21.769)$ \\
\hline
\end{tabular}

Table 4d. Multiple logistic regression analysis of the effect of total WMH volume on the likelihood of a MADRS score of $\geq 15$ at baseline

\begin{tabular}{|c|c|c|c|c|}
\hline & B & SE & $\mathrm{p}$ & $\begin{array}{l}\text { Odds ratio exp.(B) } \\
(95 \% \text { CI })\end{array}$ \\
\hline Total WMH ratio (final step) & 0.265 & 0.108 & 0.014 & $1.304(1.055-1.611)$ \\
\hline Non- $A D$ versus $A D^{*}$ (step 1$)$ & -1.040 & 0.663 & 0.116 & $0.353(0.096-1.295)$ \\
\hline
\end{tabular}

two independent variables could be used in the multiple logistic regression analyses using these cutoffs. For the same reason, these analyses could be performed only regarding the effect of the independent variables on the risk of being clinically depressed at baseline, but not at follow-up. Based on the results of previous analyses, as well as bivariate logistic regression analyses (data not shown), prior depression yes/no or AD/non-AD were entered as independent variables in stepwise multiple logistic regression analyses, together with either frontal deep WMH ratios or total WMH ratios.

Using MADRS scores of $<15$ versus $\geq 15$ as the response variable, both a higher frontal deep WMH ratio and prior depression were significant predictors of having a MADRS score of $\geq 15$. Replacing prior depression with $\mathrm{AD} /$ non-AD in the analysis, only the frontal deep $\mathrm{WMH}$ ratio remained significant. Similar findings emerged for the total WMH ratio (table $4 \mathrm{a}-\mathrm{d}$ ).

Using NPId scores of $<4$ versus $\geq 4$ as the response variable, only prior depression was significant when entered with frontal deep WMH ratios into the analysis (the validity of the model fit was uncertain, Pearson $\chi^{2}$ goodness-of-fit value $=0.000$ ). However, only higher 
Table 5a. Multiple logistic regression analysis of the effect of frontal deep WMH volume on the likelihood of a NPId score of $\geq 4$ at baseline

\begin{tabular}{llllll}
\hline & B & SE & p & $\begin{array}{l}\text { Odds ratio exp. (B) } \\
(95 \% \text { CI })\end{array}$ \\
\hline $\begin{array}{l}\text { Frontal deep WMH ratio } \\
\text { Prior depression }\end{array}$ & - & - & - & - \\
\hline
\end{tabular}

Table 5b. Multiple logistic regression analysis of the effect of frontal deep WMH volume on the likelihood of a NPId score of $\geq 4$ at baseline

\begin{tabular}{|c|c|c|c|c|}
\hline & $\mathrm{B}$ & SE & $\mathrm{p}$ & $\begin{array}{l}\text { Odds ratio exp. (B) } \\
(95 \% \mathrm{CI})\end{array}$ \\
\hline Frontal deep WMH ratio (final step) & 0.703 & 0.348 & 0.044 & $2.019(1.021-3.993)$ \\
\hline Non-AD versus $\mathrm{AD}^{*}$ (step 1$)$ & -0.542 & 0.655 & 0.408 & $0.581(0.161-2.098)$ \\
\hline
\end{tabular}

Table 5c. Multiple logistic regression analysis of the effect of total WMH volume on the likelihood of a NPId score of $\geq 4$ at baseline

\begin{tabular}{llllll}
\hline & B & SE & p & $\begin{array}{l}\text { Odds ratio exp. (B) } \\
\text { (95\% CI) }\end{array}$ \\
\hline $\begin{array}{lllll}\text { Total WMH ratio } \\
\text { Prior depression }\end{array}$ & 0.275 & 0.108 & 0.011 & $\begin{array}{l}1.317(1.066-1.628) \\
2.908(0.842-10.038)\end{array}$ \\
\hline
\end{tabular}

Table 5d. Multiple logistic regression analysis of the effect of total WMH volume on the likelihood of a NPId score of $\geq 4$ at baseline

\begin{tabular}{|c|c|c|c|c|}
\hline & B & SE & $\mathrm{p}$ & $\begin{array}{l}\text { Odds ratio exp. (B) } \\
(95 \% \mathrm{CI})\end{array}$ \\
\hline Total WMH ratio (final step) & 0.304 & 0.107 & 0.005 & $1.356(1.099-1.673)$ \\
\hline Non-AD versus $\mathrm{AD}^{*}$ (step 1$)$ & -0.348 & 0.686 & 0.612 & $0.706(0.184-2.709)$ \\
\hline
\end{tabular}

* Non-AD $=0, \mathrm{AD}=1$. MADRS: normal range 0-6.

frontal deep WMH ratios were significantly associated with NPId scores of $\geq 4$ when entered into the analysis with $\mathrm{AD} /$ non-AD. Replacing frontal deep WMH ratios with total WMH ratios, higher total WMH ratios, but not prior depression or $\mathrm{AD} /$ non-AD were significantly associated with a NPId score of $\geq 4$ (table $5 \mathrm{a}-\mathrm{d}$ ).

All the models having clinically significant depression as response variable were statistically significant, i.e. Omnibus test of model coefficients $\mathrm{p}<0.05$.

\section{Course of Depression}

The median change in MADRS scores was +1.0 . Of the 206 subjects, $47 \%$ had a MADRS score of $\geq 7$ at the 1 -year follow-up, and $12 \%$ had a MADRS score of $\geq 15$. Using a MADRS cutoff score for depression of $\geq 15,20$ out of 35 (57\%) recovered and $5 \%$ had incident depression. Baseline total and frontal deep WMH were not correlated with MADRS ( $p=0.459$ and $\mathrm{p}=0.537$, respectively) or NPId ( $\mathrm{p}=0.697$ and $\mathrm{p}=0.329$, respectively) scores at the 1 -year follow-up. 
Using cutoff scores for depression of $\geq 7$ and $\geq 1$, respectively, for MADRS and NPId, there were no significant differences in WMH ratios when comparing patients with favourable (i.e. never depressed or remission) and unfavourable (i.e. new depression or persistence) courses of depression, in remission versus persistent depression, or between patients who had never had depression versus those with persistent depression. Finally, changes between baseline and follow-up MADRS and NPId scores were not significantly correlated with WMH ratios at baseline.

However, patients with MADRS scores of $\geq 15$ at follow-up had significantly higher baseline volumes of both total and frontal deep WMH ( $p=0.012$ and $p=0.006$, respectively) than those with a MADRS score of $\leq 14$. Furthermore, patients with an unfavourable course according to MADRS scores had significantly higher volumes of both total and frontal deep $\mathrm{WMH}(\mathrm{p}=0.012$ and $\mathrm{p}=0.006$, respectively). In the NPId, this was the case only for frontal deep WMH ( $\mathrm{p}=0.047)$. There were no significant differences between patients with remission compared to those with persistent depression with respect to WMH volumes. When comparing patients with no depression and patients with persistent depression, the only significant difference was that patients with persistent depression based on the NPId had significantly higher volumes of frontal deep WMH $(\mathrm{p}=0.031)$. There were no other associations using the NPId $\geq 4$ cutoff score.

No significant associations were found between Scheltens total and frontal scores and the course of depression variables.

\section{Discussion}

\section{Main Findings}

The main finding of this study was that the volume ratios of WMH were positively and independently correlated with depression at baseline. Furthermore, the results suggest that having higher WMH volume ratios at baseline may be associated with a higher risk of having depression or an unfavourable course of depression at the 1-year follow-up.

\section{Interpretation}

The results of our study are consistent with some previous studies [3,9], and similar findings have been reported also in non-demented elderly [7, 34]. However, other studies did not find such a relationship, although this was based on a combination of psychiatric symptoms rather than a specific measure of depression [10]. Thus, our findings support the hypothesis that WMH predispose elderly individuals, with or without dementia, to developing depression. One possible mechanism is disruption of fibre tracts connecting cortical and subcortical structures involved in the regulation of mood [1].

However, our hypothesis that the prognosis of depressive symptoms in persons with mild dementia is related to the volume of WMH, and frontal deep WMH in particular, was less robustly supported. One partial explanation could be the rather low total number of patients, and consequently a low number of patients having clinically significant depression in our study, resulting in limited possibilities for multivariable statistical analysis. Moreover, cerebrovascular disease is a chronic condition that may exert its major influence early in the course of dementia, and other factors, intrinsic and extrinsic, could be more important determinants of the course of depression at later stages. These factors may include neurotransmitter changes [35], inflammation [36], physical comorbidity [37], environmental factors [38], treatment [39], and disability [6]. Of note, we recently found that worsening of depressive symptoms was associated with a more rapid cognitive decline, suggesting that the degenerative changes at this stage of the disease are the key determinants 
of depression in people with early AD and LBD [11]. Finally, the less-than-robust support for our hypothesis might be related to longitudinal changes in depression in elderly patients with dementia [40].

The observed trend towards a differential relationship between WMH and depression in $\mathrm{AD}$ versus $\mathrm{LBD}$ is most likely due to differences in statistical power, due to the small number of LBD patients.

We did, somewhat surprisingly, find almost no correlations or associations between Scheltens scores and depression variables. This is in contrast to findings in a previous study [3]. Notably, this previous study included persons with more advanced dementia (mean MMSE scores of 13.6-18.2) than our study. This discrepancy could also be due to visual scoring methods, e.g. the Scheltens scale, being less sensitive than volumetric methods [41], despite the high correlations between the results of these methods. Unlike volumetric methods, visual rating scales, such as the Scheltens scale, display ceiling effects and poor discrimination of absolute lesion volumes [41].

\section{Strengths and Limitations}

Strengths of this study include the longitudinal design and the quantitative method for measuring WMH. Limitations include the relatively low number of cases, which limits the statistical power of the study, particularly for the LBD group. Moreover, our sample was recruited from secondary care outpatient clinics, and may therefore not be representative of the general population with mild dementia. Thus, the generalisability of our findings may be limited. The change in depression was relatively small, probably due to the relatively short study period, which also limits the statistical power to detect associations with the longitudinal course of depression. A longer observation period might have increased the likelihood of finding an association between WMH and the course of depression. Another possible limitation is that depression was assessed using scale scores rather than using a structured psychiatric interview and diagnoses based on standardised criteria. The diagnosis in the majority of cases was clinical, and thus misdiagnosis cannot be excluded. However, standardised and validated scales were used, also to support the diagnosis of DLB, where the clinical diagnosis was confirmed in all 7 patients who came to autopsy [Dag Aarsland, pers. commun., 2011]. We combined patients with DLB and PDD into one LBD group. Although the two syndromes differ in the temporal sequence of symptoms and in the relative severity of cortical amyloid pathology, we consider them sufficiently similar to be combined in a study of WMH. Furthermore, patients were followed longitudinally, which also is likely to increase diagnostic accuracy. We used two different measures of depression, one observer-based (MADRS) and one carer-based (NPId). Stronger associations with WMH were found for MADRS, suggesting that this is a more sensitive measure of depression in mild dementia. Finally, since this was a naturalistic study, we could not control for possible treatment changes during the study period, which might influence the course of depression and thus mask a possible relationship between WMH and the course of depression.

\section{Clinical Implications and Future Research}

One implication of our findings is that prevention or reduction of $\mathrm{WMH}$, if possible, could lessen the burden of depression and depressive symptoms in persons with mild dementia. This might be accomplished by means of therapies directed against established risk factors for stroke and WMH, such as hypertension and diabetes [42]. Such preventive measures, which would at least have several other positive health effects, should probably be initiated in midlife [43]. The importance of such potentially preventive strategies is increased in light of recent studies suggesting that antidepressants may not be effective for depressed patients with dementia [44]. 
Further studies of the mechanisms that determine the course of depression in people with mild dementia are clearly called for. These studies should be sufficiently powered and of longer duration, as well as including patients with non-AD dementias such as LBD and vascular dementia.

\section{Acknowledgement}

This study was supported financially by Helse Stavanger HF and by grant 911694 from the Western Norway Regional Health Authority.

\section{Disclosure Statement}

None of the authors has any conflicts of interest related to the current article.

\section{References}

-1 Herrmann LL, Le Masurier M, Ebmeier KP: White matter hyperintensities in late life depression: a systematic review. J Neurol Neurosurg Psychiatry 2008;79:619-624.

-2 Breteler MM, van Swieten JC, Bots ML, Grobbee DE, Claus JJ, van den Hout JH, van Harskamp F, Tanghe HL, de Jong PT, van Gijn J, et al: Cerebral white matter lesions, vascular risk factors, and cognitive function in a population-based study: the Rotterdam study. Neurology 1994;44:1246-1252.

-3 Barber R, Scheltens P, Gholkar A, Ballard C, McKeith I, Ince P, Perry R, O'Brien J: White matter lesions on magnetic resonance imaging in dementia with Lewy bodies, Alzheimer's disease, vascular dementia, and normal aging. J Neurol Neurosurg Psychiatry 1999;67:66-72.

-4 Dufouil C, de Kersaint-Gilly A, Besancon V, Levy C, Auffray E, Brunnereau L, Alperovitch A, Tzourio C: Longitudinal study of blood pressure and white matter hyperintensities: the EVA MRI Cohort. Neurology 2001;56:921-926.

-5 Wakefield DB, Moscufo N, Guttmann CR, Kuchel GA, Kaplan RF, Pearlson G, Wolfson L: White matter hyperintensities predict functional decline in voiding, mobility, and cognition in older adults. J Am Geriatr Soc 2010;58:275-281.

-6 Teodorczuk A, Firbank MJ, Pantoni L, Poggesi A, Erkinjuntti T, Wallin A, Wahlund LO, Scheltens P, Waldemar G, Schrotter G, Ferro JM, Chabriat H, Bazner H, Visser M, Inzitari D, O'Brien JT: Relationship between baseline white-matter changes and development of late-life depressive symptoms: 3-year results from the LADIS study. Psychol Med 2010;40:603-610.

-7 Godin O, Dufouil C, Maillard P, Delcroix N, Mazoyer B, Crivello F, Alperovitch A, Tzourio C: White matter lesions as a predictor of depression in the elderly: the 3C-Dijon study. Biol Psychiatry 2008; 63:663-669.

8 O'Brien J, Ames D, Chiu E, Schweitzer I, Desmond P, Tress B: Severe deep white matter lesions and outcome in elderly patients with major depressive disorder: follow-up study. BMJ 1998;317:982-984.

-9 O’Brien J, Perry R, Barber R, Gholkar A, Thomas A: The association between white matter lesions on magnetic resonance imaging and noncognitive symptoms. Ann N Y Acad Sci 2000;903:482-489.

- 10 Modrego PJ, Rios C, Perez Trullen JM, Errea JM, Garcia-Gomara MJ, Sanchez S: The cerebrovascular pathology in Alzheimer's disease and its influence on clinical variables. Am J Alzheimers Dis Other Demen 2008;23:91-96.

-11 Fritze F, Ehrt U, Hortobagyi T, Ballard C, Aarsland D: Depressive symptoms in Alzheimer's disease and Lewy body dementia: a one-year follow-up study. Dement Geriatr Cogn Disord 2011;32:143-149.

-12 Krishnan KR, Delong M, Kraemer H, Carney R, Spiegel D, Gordon C, McDonald W, Dew M, Alexopoulos G, Buckwalter K, Cohen PD, Evans D, Kaufmann PG, Olin J, Otey E, Wainscott C: Comorbidity of depression with other medical diseases in the elderly. Biol Psychiatry 2002;52:559-588. 
-13 Starkstein SE, Jorge R, Mizrahi R, Robinson RG: The construct of minor and major depression in Alzheimer's disease. Am J Psychiatry 2005;162:2086-2093.

-14 Hurt C, Bhattacharyya S, Burns A, Camus V, Liperoti R, Marriott A, Nobili F, Robert P, Tsolaki M, Vellas B, Verhey F, Byrne EJ: Patient and caregiver perspectives of quality of life in dementia. An investigation of the relationship to behavioural and psychological symptoms in dementia. Dement Geriatr Cogn Disord 2008;26:138-146.

-15 Fritze F, Ehrt U, Sonnesyn H, Kurz M, Hortobagyi T, Nore SP, Ballard C, Aarsland D: Depression in mild dementia: associations with diagnosis, APOE genotype and clinical features. Int J Geriatr Psychiatry 2011;26:1054-1061.

-16 Aarsland D, Rongve A, Nore SP, Skogseth R, Skulstad S, Ehrt U, Hoprekstad D, Ballard C: Frequency and case identification of dementia with Lewy bodies using the revised consensus criteria. Dement Geriatr Cogn Disord 2008;26:445-452.

-17 Aarsland D, Ballard CG, Halliday G: Are Parkinson's disease with dementia and dementia with Lewy bodies the same entity? J Geriatr Psychiatry Neurol 2004;17:137-145.

-18 McKeith IG, Dickson DW, Lowe J, Emre M, O’Brien JT, Feldman H, Cummings J, Duda JE, Lippa C, Perry EK, Aarsland D, Arai H, Ballard CG, Boeve B, Burn DJ, Costa D, Del Ser T, Dubois B, Galasko D, Gauthier S, Goetz CG, Gomez-Tortosa E, Halliday G, Hansen LA, Hardy J, Iwatsubo T, Kalaria RN, Kaufer D, Kenny RA, Korczyn A, Kosaka K, Lee VM, Lees A, Litvan I, Londos E, Lopez OL, Minoshima S, Mizuno Y, Molina JA, Mukaetova-Ladinska EB, Pasquier F, Perry RH, Schulz JB, Trojanowski JQ, Yamada M: Diagnosis and management of dementia with Lewy bodies: third report of the DLB consortium. Neurology 2005;65:1863-1872.

-19 Cummings JL, Mega M, Gray K, Rosenberg-Thompson S, Carusi DA, Gornbein J: The neuropsychiatric inventory: comprehensive assessment of psychopathology in dementia. Neurology 1994;44: 2308-2314.

20 Selbaek G, Kirkevold O, Sommer OH, Engedal K: The reliability and validity of the Norwegian version of the Neuropsychiatric Inventory, nursing home version (NPI-NH). Int Psychogeriatr 2008;20: 375-382.

-21 Caputo M, Monastero R, Mariani E, Santucci A, Mangialasche F, Camarda R, Senin U, Mecocci P: Neuropsychiatric symptoms in 921 elderly subjects with dementia: a comparison between vascular and neurodegenerative types. Acta Psychiatr Scand 2008;117:455-464.

-22 Montgomery SA, Asberg M: A new depression scale designed to be sensitive to change. Br J Psychiatry 1979;134:382-389.

23 Snaith RP, Harrop FM, Newby DA, Teale C: Grade scores of the Montgomery-Asberg Depression and the Clinical Anxiety Scales. Br J Psychiatry 1986;148:599-601.

-24 Leontjevas R, van Hooren S, Mulders A: The Montgomery-Asberg Depression Rating Scale and the Cornell Scale for Depression in Dementia: a validation study with patients exhibiting early-onset dementia. Am J Geriatr Psychiatry 2009;17:56-64.

-25 Muller-Thomsen T, Arlt S, Mann U, Mass R, Ganzer S: Detecting depression in Alzheimer's disease: evaluation of four different scales. Arch Clin Neuropsychol 2005;20:271-276.

-26 Conwell Y, Forbes NT, Cox C, Caine ED: Validation of a measure of physical illness burden at autopsy: The Cumulative Illness Rating Scale. J Am Geriatr Soc 1993;41:38-41.

27 Firbank MJ, Lloyd AJ, Ferrier N, O’Brien JT: A volumetric study of MRI signal hyperintensities in late-life depression. Am J Geriatr Psychiatry 2004;12:606-612.

$\checkmark 28$ Firbank MJ, Minett T, O’Brien JT: Changes in DWI and MRS associated with white matter hyperintensities in elderly subjects. Neurology 2003;61:950-954.

-29 Scheltens P, Barkhof F, Leys D, Pruvo JP, Nauta JJ, Vermersch P, Steinling M, Valk J: A semiquantative rating scale for the assessment of signal hyperintensities on magnetic resonance imaging. J Neurol Sci 1993;114:7-12.

-30 Cole MG, Dendukuri N: Risk factors for depression among elderly community subjects: a systematic review and meta-analysis. Am J Psychiatry 2003;160:1147-1156.

-31 Solhaug HI, Romuld EB, Romild U, Stordal E: Increased prevalence of depression in cohorts of the elderly: an 11-year follow-up in the general population - the Hunt Study. Int Psychogeriatr 2012;24: 151-158.

-32 Cohen LS: Gender-specific considerations in the treatment of mood disorders in women across the life cycle. J Clin Psychiatry 2003;64(suppl 15):18-29. 
-33 Chang-Quan H, Xue-Mei Z, Bi-Rong D, Zhen-Chan L, Ji-Rong Y, Qing-Xiu L: Health status and risk for depression among the elderly: a meta-analysis of published literature. Age Ageing 2010;39:23-30.

34 Teodorczuk A, Firbank MJ, Pantoni L, Poggesi A, Erkinjuntti T, Wallin A, Wahlund LO, Scheltens P, Waldemar G, Schrotter G, Ferro JM, Chabriat H, Bazner H, Visser M, Inzitari D, O’Brien JT: Relationship between baseline white-matter changes and development of late-life depressive symptoms: 3-year results from the LADIS study. Psychol Med 2009;1-8.

-35 Korczyn AD, Halperin I: Depression and dementia. J Neurol Sci 2009;283:139-142.

-36 Leonard BE: Inflammation, depression and dementia: are they connected? Neurochem Res 2007;32: 1749-1756.

-37 Alexopoulos GS: Depression in the elderly. Lancet 2005;365:1961-1970.

- 38 Schoevers RA, Beekman AT, Deeg DJ, Geerlings MI, Jonker C, Van Tilburg W: Risk factors for depression in later life; results of a prospective community based study (AMSTEL). J Affect Disord 2000;59:127-137.

-39 Thompson S, Herrmann N, Rapoport MJ, Lanctot KL: Efficacy and safety of antidepressants for treatment of depression in Alzheimer's disease: a metaanalysis. Can J Psychiatry 2007;52:248-255.

-40 Wetzels R, Zuidema S, Jansen I, Verhey F, Koopmans R: Course of neuropsychiatric symptoms in residents with dementia in long-term care institutions: a systematic review. Int Psychogeriatr 2010; 22:1040-1053

-41 van Straaten EC, Fazekas F, Rostrup E, Scheltens P, Schmidt R, Pantoni L, Inzitari D, Waldemar G, Erkinjuntti T, Mantyla R, Wahlund LO, Barkhof F: Impact of white matter hyperintensities scoring method on correlations with clinical data: the LADIS study. Stroke 2006;37:836-840.

$\checkmark 42$ Ovbiagele B, Saver JL: Cerebral white matter hyperintensities on MRI: current concepts and therapeutic implications. Cerebrovasc Dis 2006;22:83-90.

-43 de Toledo Ferraz Alves TC, Ferreira LK, Busatto GF: Vascular diseases and old age mental disorders: an update of neuroimaging findings. Curr Opin Psychiatry 2010;23:491-497.

-44 Banerjee S, Hellier J, Dewey M, Romeo R, Ballard C, Baldwin R, Bentham P, Fox C, Holmes C, Katona C, Knapp M, Lawton C, Lindesay J, Livingston G, McCrae N, Moniz-Cook E, Murray J, Nurock S, Orrell M, O’Brien J, Poppe M, Thomas A, Walwyn R, Wilson K, Burns A: Sertraline or mirtazapine for depression in dementia (HTA-SADD): a randomised, multicentre, double-blind, placebocontrolled trial. Lancet 2011;378:403-411. 